\title{
Designing low-carbon power systems for Great Britain in 2050 that are robust to the spatiotemporal and inter-annual variability of weather
}

\author{
Marianne Zeyringer, James Price, Birgit Fais, Pei-Hao Li, Ed Sharp
}

\begin{abstract}
The design of cost effective power systems with high shares of variable renewable energy technologies (VREs) requires a modelling approach that simultaneously represents the whole energy system combined with the spatiotemporal and inter-annual variability of VREs. Here we soft-link a long term energy system model, that explores new energy systems configurations from years to decades, with a high spatial and temporal resolution power system model that captures VRE variability from hours to years. Applying this methodology to Great Britain for 2050, we find that VRE focused power system design is highly sensitive to the inter-annual variability of weather and that planning based on a single year can lead to operational inadequacy and failure to meet long term decarbonisation objectives. However, some insights do emerge that are relatively stable to weather year. Reinforcement of the transmission system consistently leads to a decrease in system costs while electricity storage and flexible generation, needed to integrate VREs into the system, are generally deployed close to demand centres.
\end{abstract}

The UNFCCC Paris agreement sets out the goal of limiting global average surface temperature rise to well below $2^{\circ} \mathrm{C}$ and to pursue efforts to limit it to $1.5^{\circ} \mathrm{C}$ above pre-industrial levels ${ }^{1}$. Consequently, the world is looking to clean, renewable energy solutions ${ }^{2}$ : transforming the energy system ${ }^{3}$ from being carbon intensive and inefficient ${ }^{4}$ to deeply decarbonised ${ }^{5}$, highly efficient ${ }^{4}$ and to a large extent renewable ${ }^{5}$. The power sector is leading the decarbonisation charge 6 and by 2050 low or zero carbon electricity could expand to become the dominant form of energy supply7. Renewable energy systems are characterised by the spatial and temporal variability of supply and critics therefore point to the potentially high integration costs ${ }^{2}$. Important methods to manage renewable intermittency are to integrate different renewable technologies into the system (technological diversity) and to take advantage of the fact that contemporaneous weather conditions can differ from one location to the next by spreading VRE deployment over a large geographical area (spatial diversity). By combining VRE production from different sources at different locations the variance in their output can be significantly reduced ${ }^{8,9}$. To facilitate such a system, the existing high voltage transmission network has to be reinforced or expanded. Electricity storage ${ }^{10,11}$, flexible generation ${ }^{10}$ and demand side measures ${ }^{12}$ are other options to manage the intermittency of VREs.

To provide detailed insights into the transition to a power system with high shares of VREs, we need to combine long-term planning with a representation of the spatial and temporal variability of VREs, including the inter-annual characteristics of VRE production and their integration options. Energy systems models (ESMs) allow us to: understand trade-offs between different sectors as well as the necessary mitigation burden placed on the power sector in order to reach a given carbon target; and consider investment pathways of technologies, i.e. build rates of technologies, and technology choice in previous years ${ }^{13}$. ESMs trade off spatial and temporal resolution to provide long-term pathways for the entire energy system but it has been shown that the level of spatial ${ }^{14}$, temporal ${ }^{15,16}$ and technical ${ }^{15}$ detail 
affects results in systems with a considerable amount of VREs ${ }^{17}$. Broadly speaking, in the literature there are two main approaches taken to address the challenge of modelling long-term energy systems with significant shares VREs. First, the improvement of the temporal ${ }^{18-21}$ resolution or time slice representation ${ }^{13,22}$, spatial resolution ${ }^{14}$, technical detail23 or VRE representation via a stochastic approach ${ }^{24}$ in ESMs. Second, other studies recognise that a combination of models can better represent the longterm integration of variable renewable energy sources into the power system: Refs ${ }^{25-27}$ soft-link an ESM to a temporally detailed power system model (PSM), $\operatorname{ref}^{28}$ hard-links a long-term model with a dispatch model (for a number of representative days per year), ref $^{29}$ combines a deterministic planning optimization module with a Monte Carlo simulation of system operation, ref ${ }^{30}$ links a bottom-up accounting framework to a high resolution PSM. It is worth noting that temporally and spatially explicit PSMs ${ }^{31-38}$, which allow for a good representation of VREs, have been developed but don't provide whole energy system consistency. Further, by averaging multiple years or using a single weather year most of these studies neglect the inter-annual variability of weather. Ref ${ }^{35}$ does study multiple years but only at a daily timestep and ref ${ }^{39}$ captures the effect of inter-annual variability on VRE generation but not its optimal spatial deployment pattern. The most comprehensive approaches regarding time resolution and inter-annual variability are refs 38,40 in which the system is optimized over several weather years individually but not over the contiguous time series.

In this study we combine the following key aspects which are important in understanding the design of future energy systems with high shares of VREs and ultimately achieve long term decarbonisation targets: a representation of the inter-annual variability of weather and its effect on system planning and VRE supply; the spatial and temporal detail necessary to account for differences in VRE output and timing of production, demand and infrastructure; modelling of the trade-offs and interaction of different VREs integration options; and a whole energy systems view to consider the electrification of other sectors and an internally consistent assessment of the mitigation burden placed on the electricity sector. We demonstrate this by using a modelling approach that soft-links a long-term ESM (UKTM) to a high spatial and temporal resolution PSM (highRES) (see Methods). We apply our modelling approach to Great Britain (GB), a country with limited interconnection (7\% of peak demand) and where national energy security is a high public and political priority ${ }^{41}$. Our results show that the inter-annual variability of weather substantially affects the planning and operation of power systems with medium and, in some respects even more so, high shares of VREs but consistent patterns do emerge. If additional flexibility (e.g. on the demand side) is unavailable, we find that systems planned on the basis of one weather year can lead to operational inadequacy and failure to meet long term carbon targets placed on the sector as part of the decarbonisation of the whole energy system.

\section{Soft-linking modelling approach}

Our modelling approach soft-links a long-term ESM (UKTM) to a high spatial and temporal resolution PSM (highRES). For details, see Methods. Applying this approach to Great Britain (GB), we use the ESM to develop internally consistent, whole energy system scenarios that both meet the UK's Climate Change Act $2008^{42}$, i.e. a reduction of greenhouse gas emissions of $80 \%$ relative to 1990 levels by 2050, and have high penetration of VREs. The ESM sets the boundaries on the electricity system (i.e. total electricity demand, generation capacities and $\mathrm{CO}_{2}$ grid intensity constraint) as an input to the PSM which uses ten years of weather data to capture and explore the inter-variability of weather conditions. In our methodology we aim at a balanced approach between spatiotemporal resolution, temporal coverage (i.e. how many weather years we consider) and technical detail (i.e. focusing on system adequacy and using a simplified grid representation). We give the PSM transmission grid extension, storage and flexible gas generation as VRE integration options. We choose not to include demand side measures as the large scale 
implementation in the domestic and non-domestic sector and further uptake for industry are uncertain ${ }^{12,43,44}$ due to the inherent challenges around behaviour change facing non cost-barriers ${ }^{33,45}$ and as a result studies and data on costs are limited ${ }^{46}$, even more so from a spatial distribution perspective (see Supplementary Note 9). However, like the system operator National Grid in its modelling ${ }^{47}$ we follow the recommendation ${ }^{48}$ by the UK government (Office of Gas and Electricity Markets, Ofgem; Department for Business, Energy and Industrial Strategy, BEIS) and implement load shedding at a cost set to the Value of Lost Load of $6000 f / \mathrm{MWh}$.We use the PSM to study the sensitivity of system planning and operation due to inter-annual weather variability by using ten different weather years to drive VRE production. We also compare these results to when our PSM considers a 10-year continuous time series.

\section{LCOE, emissions and capacities of VRE integration options}

Table 1 shows the power system designed by the ESM for 2050 for a scenario with 50\% (50VRE) and 80\% (80VRE) of generation from VREs.

Table 1 Historic and 2050 modelled power system characteristics

\begin{tabular}{|c|c|c|c|}
\hline Parameter & 2015 & 2050 50VRE & 2050 80VRE \\
\hline Gas Capacity (GW) & 32 & decided by highRES & decided by highRES \\
\hline Coal Capacity (GW) & 21 & 0 & 0 \\
\hline Nuclear Capacity (GW) & 9 & 34 & 12.4 \\
\hline Solar PV Capacity (GW) & 10 & 44 & 50 \\
\hline Onshore Wind Capacity (GW) & 9 & 32 & 27 \\
\hline Offshore Wind Capacity (GW) & 5 & 39 & 68 \\
\hline Biomass Capacity (GW) & 5 & 7 & 0.4 \\
\hline Interconnection Capacity (GW) & 5 & 6 & 3.6 \\
\hline Storage Capacity $(\mathrm{GW})$ & 3 (pump storage) & decided by highRES & decided by highRES \\
\hline Hydropower Capacity (GW) & 2 & 2 & 1.6 \\
\hline Other Capacity (GW) & 1 & 0.5 & 0.5 \\
\hline Electricity demand (GWh) & 358,363 & 516,882 & 416,757 \\
\hline \multicolumn{4}{|l|}{${ }^{a}$ ref. 69} \\
\hline \multicolumn{4}{|l|}{ b ref. 70} \\
\hline \multicolumn{4}{|l|}{ c ref. 71} \\
\hline \multicolumn{4}{|l|}{ d ref. 72} \\
\hline \multicolumn{4}{|l|}{ e ref. 73} \\
\hline $\begin{array}{l}\text { 50VRE and 80VRE scenarios } \\
\text { are based on UKTM unless } \\
\text { otherwise indicated }\end{array}$ & & & \\
\hline
\end{tabular}

For each scenario we study two different cases in highRES: optimising all three flexibility options (allflex); and optimising flexible generation and storage only and we fix the transmission network to its 2015 capacities (flex+store). We differ between these two scenarios as, on the one hand, transmission line extension can take decades from conception to completion, with the planning system being a 
fundamental barrier ${ }^{49}$, yet on the other hand it can unlock the benefits of greater spatial diversification. For each scenario and case, we allow the model to make investment and operational decisions based on one year of weather data at a time and 10 years simultaneously, i.e. system capacities and dispatch are planned over snapshots of 8760 hours ( 8784 hours for leap years) in the former and 87648 hours in the latter. This allows us to analyse the difference in optimal system configurations when planning for a single weather year and when considering the full variability of 10 years.

It is worth noting that for our system, in terms of generation, nuclear power functions as base load with VRE generation fluctuating on top of that base to meet demand with excess production either being sent to permit the daily cycling of storage or curtailed, of low VRE generation which are covered by flexible natural gas generation (see Supplementary Figures 12-16).

Figure 1 shows the distribution of the levelised cost of electricity (LCOE) and grid $\mathrm{CO}_{2}$ intensity in our individual year runs, i.e. one year of weather data at a time, and the runs that consider all 10 weather years simultaneously for both our VRE generation scenarios. These results demonstrate that inter-annual weather variability leads to a spread in system LCOE which is seen to increase at higher renewable penetration. That is, "poor" weather years lead to greater flexibility costs both in terms of capacity and utilisation while the opposite is true for "good" weather years. This sensitivity increases with a greater share of VRE.

For both scenarios, the allflex case has significantly (KS test, p-value $<0.01$; here after this is our definition of a significant result) lower LCOE than the flex+store, indicating this result is robust to the weather variability sampled here. In the former, greater spatial diversification allows sites with higher output and/or a favourable timing of production to be chosen leading to lower overall costs. This reduction in LCOE is seen to be greater for higher shares of VRE, underscoring how cost effective spatial diversification becomes as VRE penetration grows. Finally, the runs considering 10 weather years simultaneously are found to be costlier than the median of the individual years. This is because the system designed for the full 10 years is suboptimal for each individual year leading to higher investment and operation costs and, in the right panel, higher grid $\mathrm{CO}_{2}$ intensity.

Figure 1 also shows that system $\mathrm{CO}_{2}$ intensity changes based on weather year due to varying utilisation of flexible fossil generation. At higher VRE penetration, this generation often reaches the $\mathrm{CO}_{2}$ intensity limit required for the electricity system to be consistent with the $80 \%$ GHG reduction target applied to the whole energy system. Once more, in the vast majority of years grid intensity is significantly lower ( $\mathrm{p}$-value $<0.01$ ) in the allflex 50VRE generation runs than that scenarios flex +store case. Here, we can infer that a spatially diversified VRE portfolio leads to a lower utilisation of flexible generation.

The key point to take from Figure 1 is that inter-annual weather variability is shown to drive a substantial spread in key system metrics and, in the case of LCOE, this sensitivity increases with higher VRE penetration. 

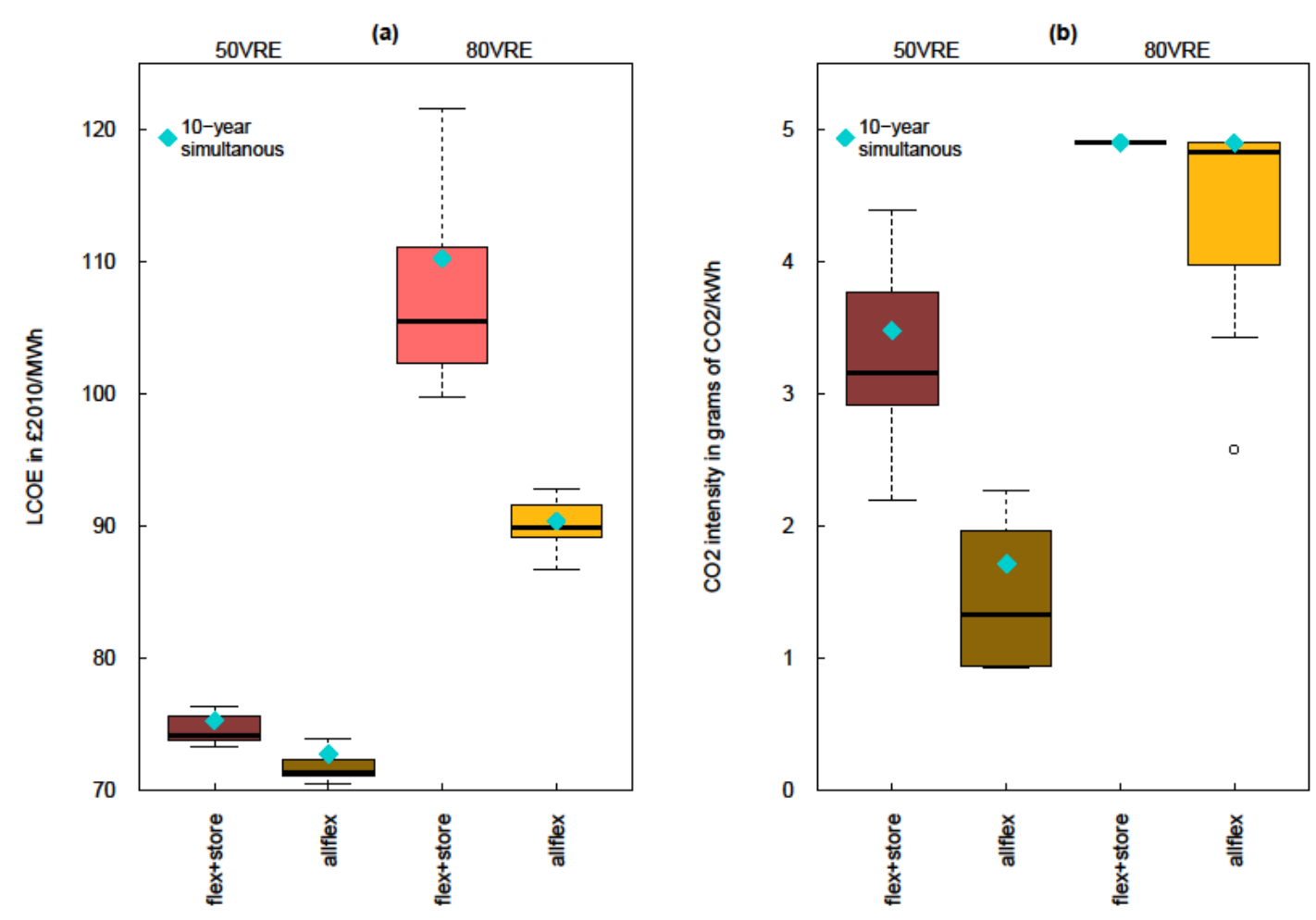

Figure 1 Distribution of LCOE and $\mathrm{CO}_{2}$ emissions for both scenarios and cases using the ten different weathers years

(a) Boxplots show the spread in LCOE over the 10 weather years. Dark/light red for the flex+store case 50VRE/80VRE scenarios respectively; dark/ light yellow for the allflex case $50 \mathrm{VRE} / 80 \mathrm{VRE}$ scenarios respectively.

(b) Boxplots show the spread in $\mathrm{CO}_{2}$ emissions over the 10 weather years. Dark/ light red for the flex+store case 50V RE/80VRE scenarios respectively; dark/ light yellow for the allflex case 50V RE/80V RE scenarios respectively.

For the boxplots the back line in the middle of the box represents the median. The box spans the first quartile to the third quartile (the interquartile range). The whiskers extend up to 1.5 times the interquartile range from the top or bottom of the box to the furthest datum within that distance. Data beyond that distance are represented as points (outliers). For (a) and (b) cyan diamonds show the results from the runs that consider 10 years simultaneously. 
(a)
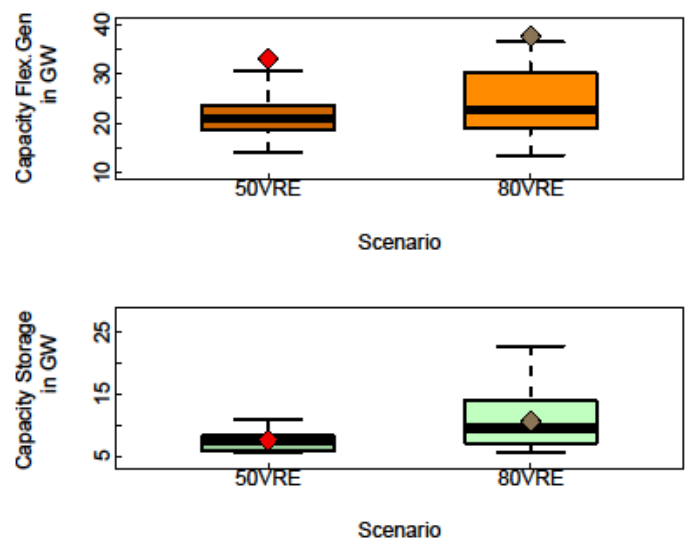

Scenario

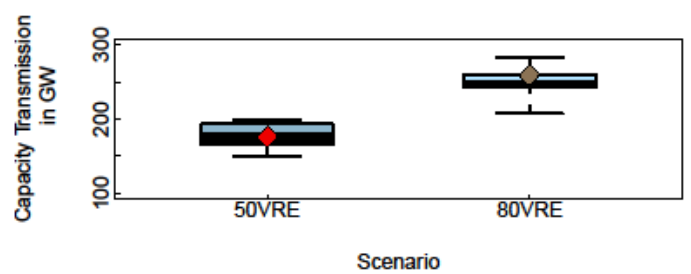

(b)
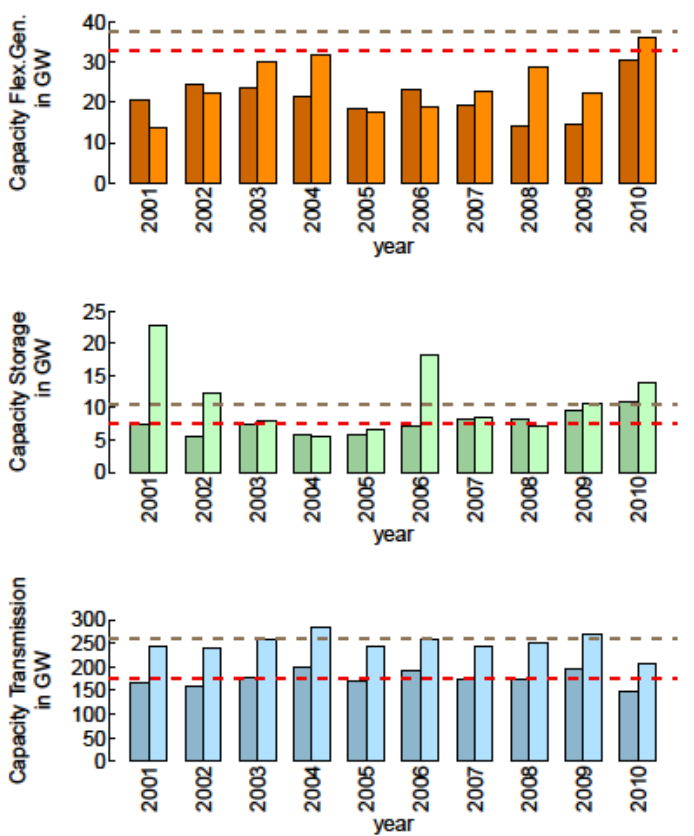

- 10-year simultaneous 50VRE capacity

- - 10-year simultaneous 50VRE capacity

10-year simultaneous 80VRE capacity

- - 10-year simultaneous 80VRE capacity

Figure 2 National installed capacities of the three VRE integration options for the $50 \mathrm{VRE}$ and $80 \mathrm{VRE}$ scenarios allflex case

(a) Orange/green/blue boxplots represent the flexible generation/storage/transmission capacity for the 10 weather years; dark orange/green/blue are for the $50 \mathrm{VRE}$ and light orange/green/blue are for the $80 \mathrm{VRE}$ scenario.

(b) Orange/green/blue bar charts represent the flexible generation/storage/transmission capacity per year; dark, orange/green/blue are for the 50V RE and light orange/green/blue are for the 80V RE scenario.

For (a) and (b) red/brown diamonds and dashed lines show the results for the 10-year simultaneous run for the 50VRE and 80VRE scenario respectively.

For the boxplots the back line in the middle of the box represents the median. The box spans the first quartile to the third quartile (the interquartile range). The whiskers extend up to 1.5 times the interquartile range from the top or bottom of the box to the furthest datum within that distance.

Figure 2 shows the total national installed capacity of the three flexibility options considered here for both our scenarios in the allflex case (flexstore case in Supplementary Figure 18). In 50VRE, flexible generation and storage vary by $\sim \pm 40 \%$ while transmission capacities are considerably less sensitive to weather year, varying by $\sim \pm 15 \%$ and showing a 70\% increase relative to 2015 levels. Figure 2 also shows that the capacity credit of VRE, i.e. the amount of firm capacity replaced per unit installed, is highly dependent on weather year (see Supplementary Table 8 for the calculation and capacity credit per scenario and year).

The 80VRE scenario has a greater spread of natural gas and storage capacities than 50VRE and, while the distributions are not statistically different between the scenarios, it is worth remembering that the total demand is lower in the former and so the installed capacity per unit demand is greater. In addition, transmission line capacities are significantly higher in 80VRE, indicating that reinforcement remains a consistent, cost effective option across the weather years as VRE shares increase.

In the 10 weather simultaneous cases we see that the capacity of flexible generation is higher than any of the individual years. This occurs for two reasons, its capacity is set by the hours at the peak in the 
decadal residual load time series combined with the fact that the 10 year system design is sub optimal for each individual year. The capacities of storage and transmission do not see such a substantial increase. For storage, this is because it is more cost effective for it to be cycled daily to cover diurnal peak load and for low capital cost, high marginal cost gas to be used to cover all-time peaks in the demand time series and periods of low storage availability due to low VRE output. For transmission, the relatively minor increase is caused by the system design being sub optimal for each individual year.

\section{Spatial deployment of VREs and VRE integration options}

In this section, we show where the highRES model locates VRE capacities and VRE integration options. Figure 3 shows the locations of the zones in Figures 4 and 5.

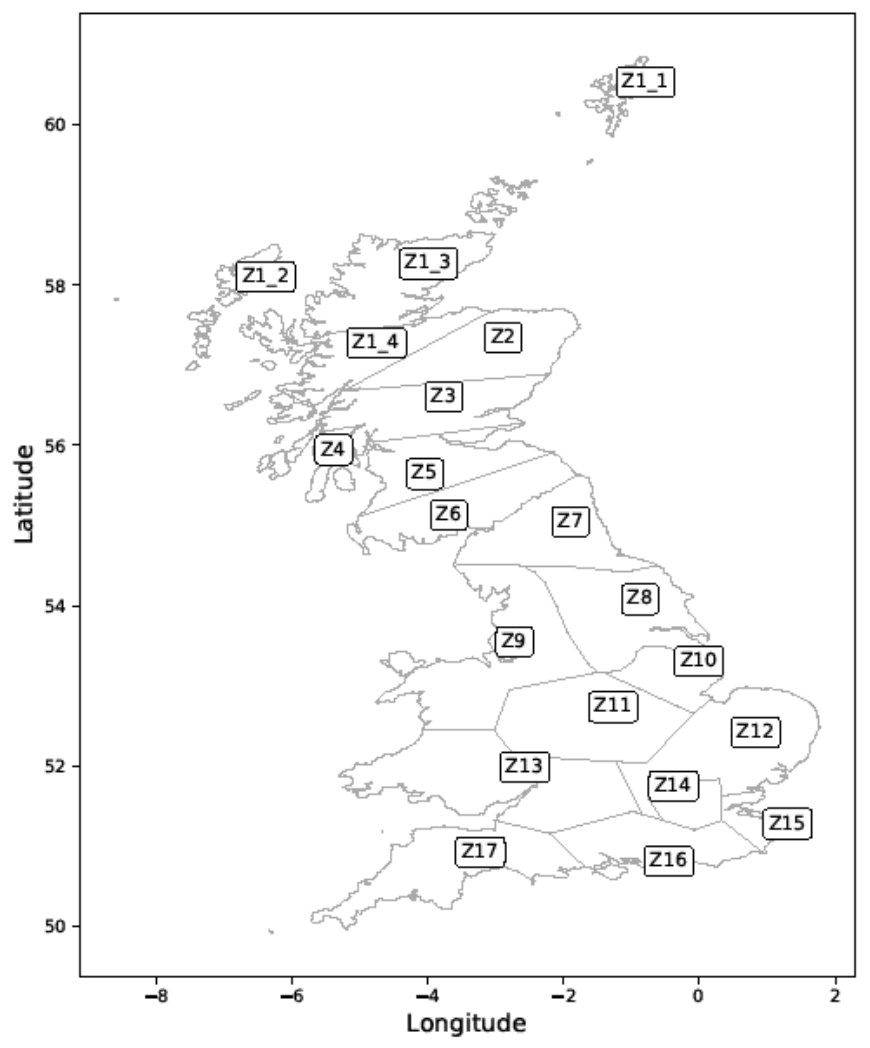

Figure 3 Location of zones 

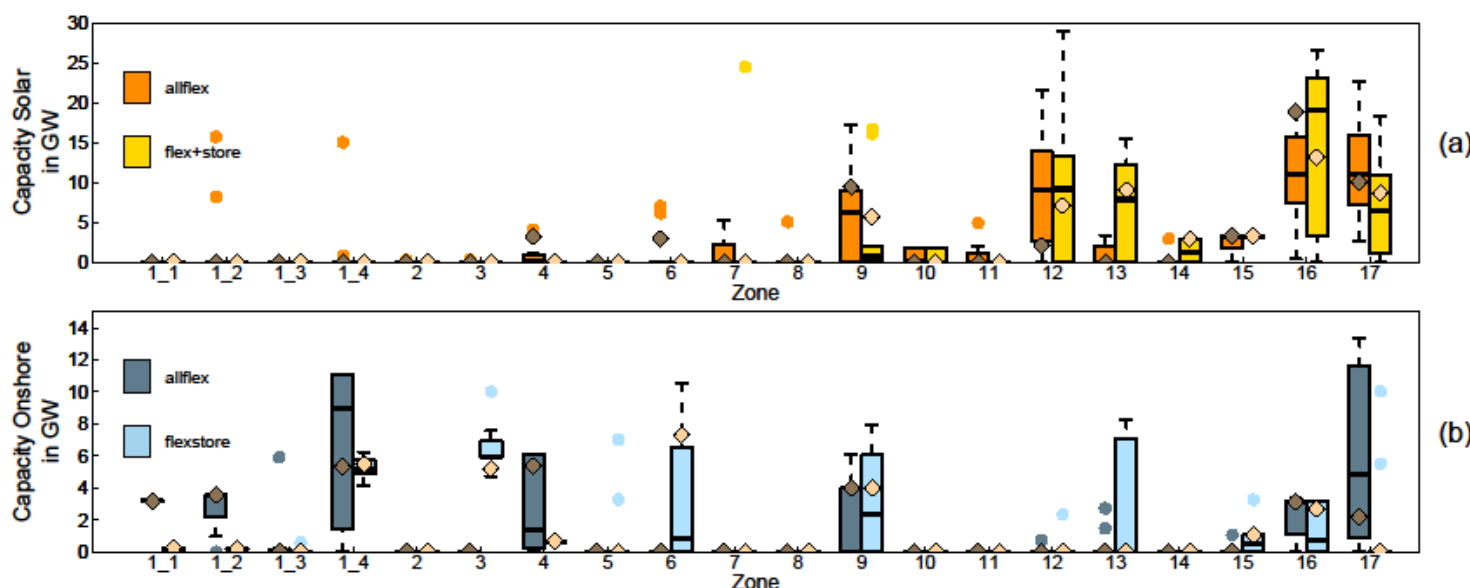

(b)

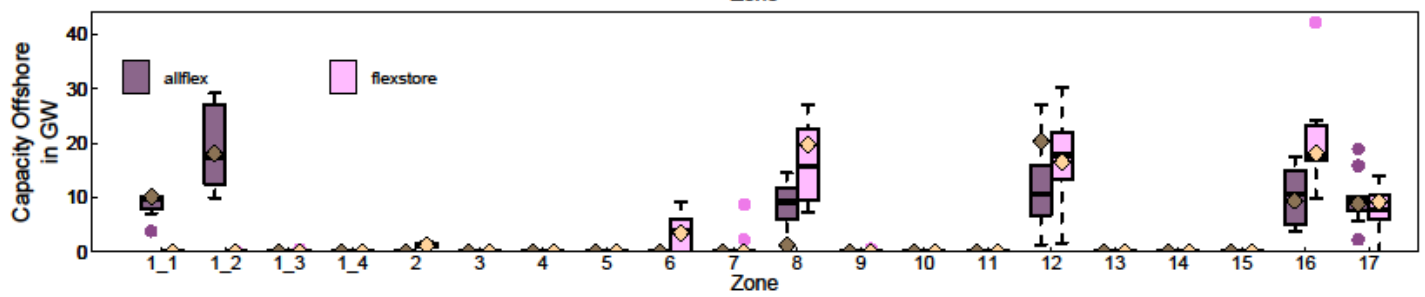

(c)

- 10-year simultaneous 50VRE

$\diamond \quad$ 10-year simultaneous 80VRE

Figure 4 Location and capacities of $\mathrm{V}$ REs for the $80 \mathrm{VRE}$ scenario in allflex and flexstore cases

(a) Solar capacity per zone: orange boxplots represent the 10 weather years allflex case, yellow boxplots resent the 10 weather years flex+store case.

(b) Onshore wind capacity per zone: dark blue boxplots represent the 10 weather years allflex case, light blue boxplots resent the 10 weather years flex+store case.

(c) Offshore wind capacity per zone: dark purple boxplots represent the 10 weather years allflex case, light purple boxplots resent the 10 weather years flex+store case.

For (a), (b) and (c) brown/ beige diamonds represent the capacities resulting from the 10-year simultaneous run allflex and flexstore cases respectively. See Figure 3 for definitions of the zones.

For the boxplots the back line in the middle of the box represents the median. The box spans the first quartile to the third quartile (the interquartile range). The whiskers extend up to 1.5 times the interquartile range from the top or bottom of the box to the furthest datum within that distance. Data beyond that distance are represented as points (outliers). Figure 4 shows the locations of the VRE capacities for the $80 \mathrm{VRE}$ scenario in the allflex and flex store cases (for 50VRE scenario see Supplementary Figure 17). We find that in the 80 VRE scenario solar energy is mainly deployed in the South where the highest capacity factors are. Onshore wind is consistently located in Scotland, although without additional transmission capacity it is not deployed on Shetland or the Outer Hebrides, and West, South and South-West of England. Offshore wind is placed around the North-Sea, the South and SouthWest and in the allflex scenario it is additionally located in Scotland. In both scenarios, wind is spread around the country in sites that balance high average capacity factors with a system beneficial timing of production. However, in the flex + store case the model is constrained by the current transmission line capacity and thus chooses locations further South, closer to demand.

While consistent deployment patterns emerge, the precise amount of capacity located in each zone is highly sensitive to the inter-annual variability of the weather data. Furthermore, for PV and, to a lesser extent wind, capacity deployment outliers are observed in some years. In the case of solar, this occurs because the transmission system is consistently reinforced to move wind generated electricity down from Scotland and Northern England (see Figure 5) to demand centres in the South. Given this 
large transmission capacity and the relatively small annual capacity factor differences between Southern England ( 0.11-0.12) and Scotland ( 0.09-0.1), for some years it becomes optimal to build solar further North than would be expected. Hence the small number of solar outliers in the flex + store results.

While the installed capacity in a zone is certainly correlated with that zones annual capacity factor, Supplementary figures 19-29 clearly show that the model balances high capacity factors with a system beneficial timing of production (i.e. spatial diversification). When running all ten years at once, both cases follow similar deployment patterns to their respective individual year runs albeit without choosing any outlier locations. The key point here is that the deployment variability in the individual year runs is seen to capture the locations and capacities chosen by the 10-year simultaneous run for both scenarios. However, we note that the capacities deployed in the full decadal runs often do not match the median levels from the box plots shown in Figure 4.
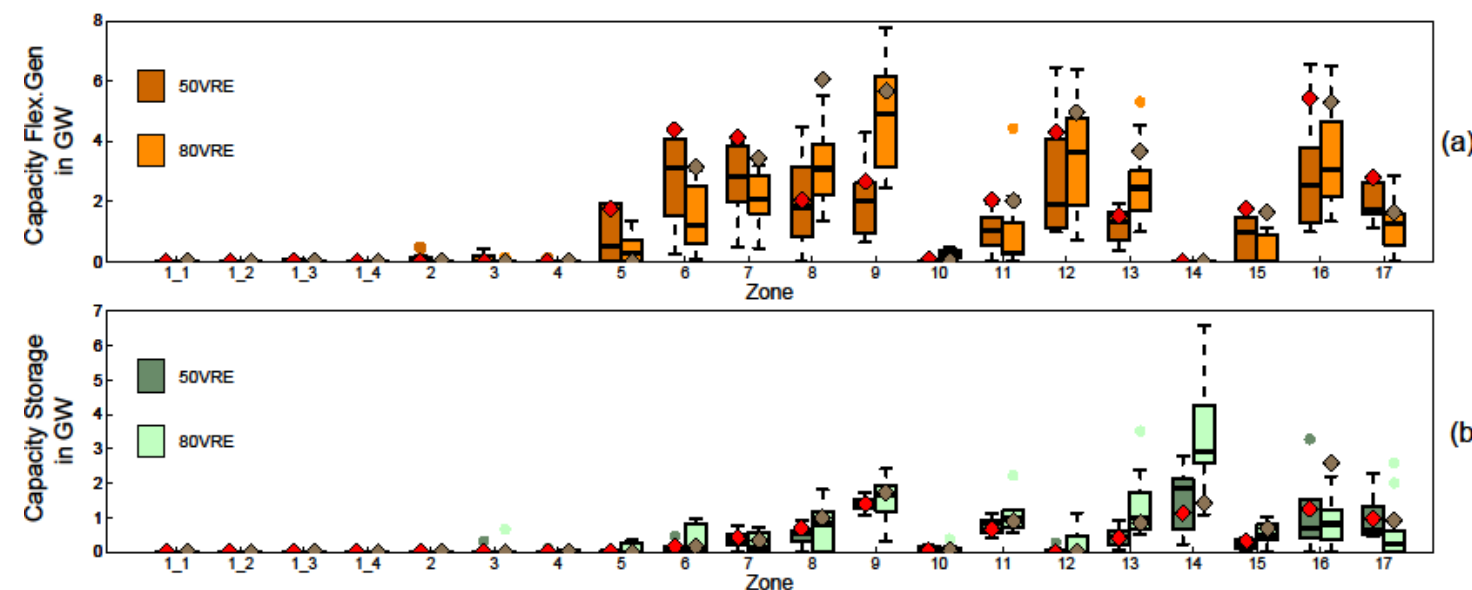

(b)

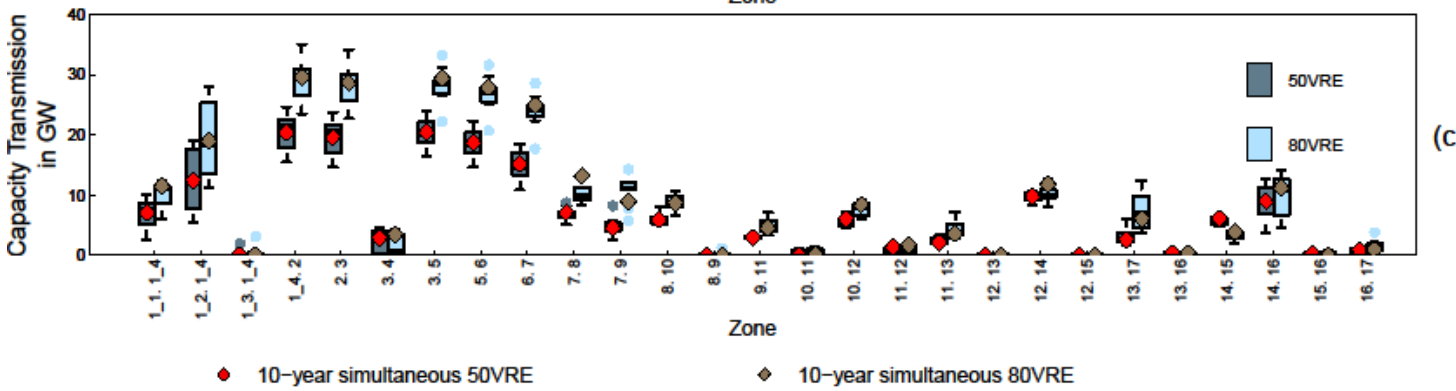

Figure 5 Location and capacities of $V R E$ integration options for the $80 \mathrm{VRE}$ and $50 \mathrm{VRE}$ scenarios allflex case

(a) Flexible generation capacity per zone: dark orange boxplots represent the 10 weather years 50V RE scenario, light orange boxplots resent the 10 weather years $80 \mathrm{~V} \mathrm{RE}$ scenario.

(b) Storage capacity per zone: dark, green boxplots represent the 10 weather years 50V RE scenario, light green boxplots resent the 10 weather years $80 \mathrm{VRE}$ scenario.

(c) Transmission capacity per zone: dark blue boxplots represent the 10 weather years $50 \mathrm{VRE}$ scenario, light purple boxplots represent the 10 weather years $80 \mathrm{~V}$ RE scenario.

For (a), (b) and (c) red/ brown diamonds represent the capacities resulting from the 10-year simultaneous run $50 \mathrm{VRE}$ and 80VRE respectively. See Figure 3 for definitions of the zones.

For the boxplots the back line in the middle of the box represents the median. The box spans the first quartile to the third quartile (the interquartile range). The whiskers extend up to 1.5 times the interquartile range from the top or bottom of the box to the furthest datum within that distance. Data beyond that distance are represented as points (outliers). 
Figure 5 compares the placement of VRE integration options in the two scenarios for the allflex case. We see that the model places storage mainly around demand centres (with the largest amount of storage typically located in London) and not close to installed VRE capacity (compare Figure 4 and Figure 5 ) in the North but the exact location is dependent on the weather year. As one would expect flexible generation is installed close to demand. The transmission network undergoes a consistent, sizable reinforcement to bring electricity from Scotland to the South, Southern England to London and Devon/Cornwall to South Wales. When combined with Figure 2, this demonstrates that grid reinforcement at the national level is robust to inter-annual weather variability and, as such, is likely to be a low risk investment option in systems with significant VRE shares. While the spatial pattern is very similar, the level of reinforcement tends to be consistently greater in the 80VRE scenario. Again, the variability of the 10 individual years mostly captures the variability in the 10-year simultaneous run.

\section{Basing system design on single weather years}

Given that we have demonstrated the sizable impact the inter-annual variability of weather has on power system design, next we test how planning for a single year affects system operation in other years. For each VRE generation scenario we identify the most weather sensitive allflex system configuration (see Methods; 2008 for 50VRE and 2005 for 80VRE) and run it with the remaining nine weather/demand years. From Figure 6 we see that both our scenarios have hours where supply does not match demand. The most weather sensitive $80 \mathrm{VRE}$ system has over $5 \%$ of hours with unmet demand ranging up to $33 \mathrm{GW}$, a substantially higher fraction than the 50VRE case. This is due both to the formers greater sensitivity to weather and the interaction of that sensitivity with the grid $\mathrm{CO}_{2}$ intensity limit applied to the system. Here we clearly see that planning VRE focused systems based on a single weather year can lead to operational inadequacy and failure to meet long term carbon reduction targets (see Supplementary Figure 30) unless further system flexibility is available (e.g. on the demand side).

(a) $50 \%$ VREGEN (total unmet hours $=333$ out of 78864 )

(b) $80 \%$ VREGEN (total unmet hours $=4465$ out of 78888 )
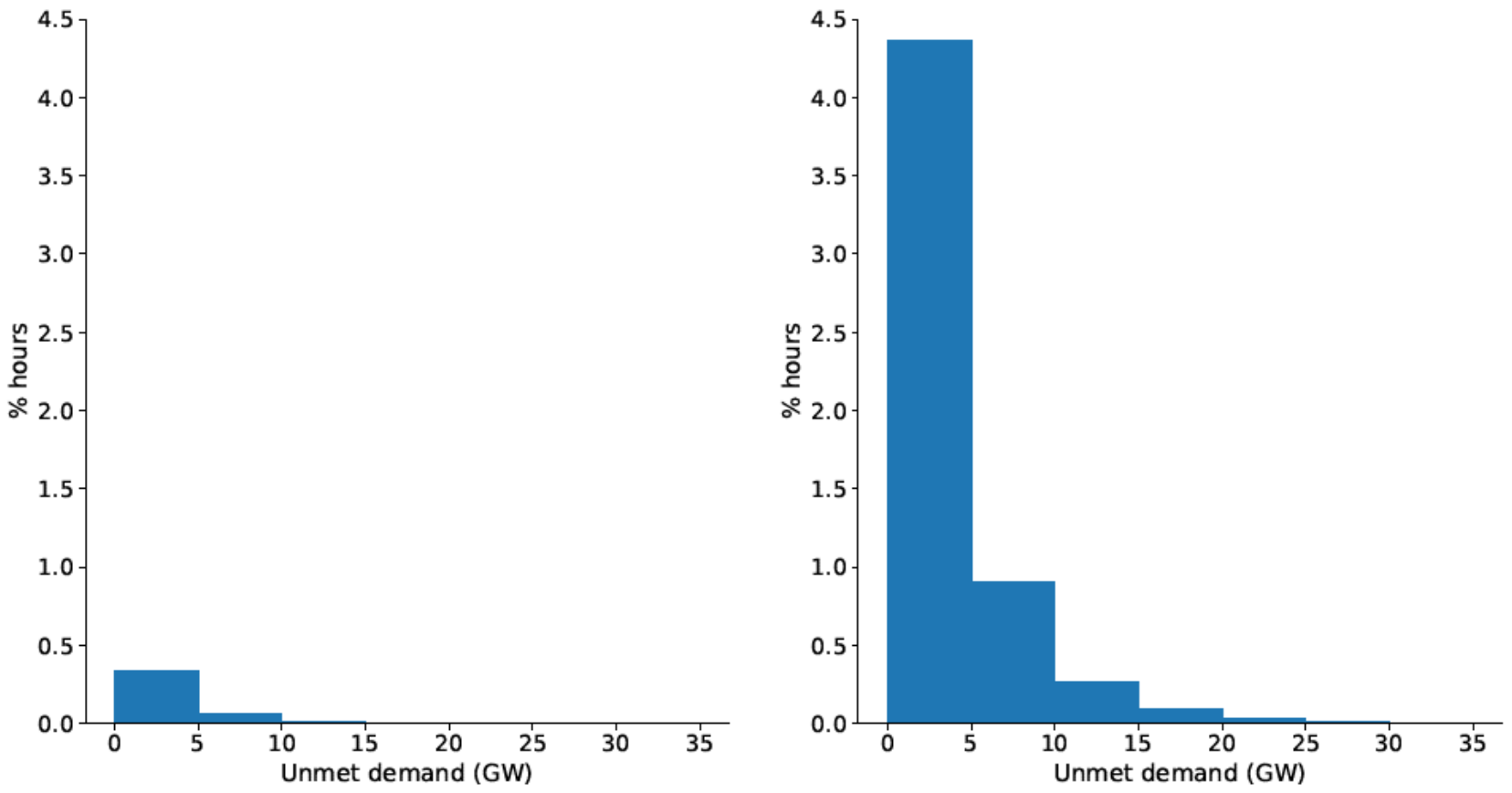
Figure 6 Unmet demand hours as a result of planning the power system based on a single weather year

The percentage of hours where supply and demand do not match as a function of the mount of unmet demand during that hour when planning the power system based on (a) the weather year 2008 for $50 \mathrm{VRE}$ and (b) the weather year 2005 for $80 \mathrm{VRE}$ and then driving the system with the remaining 9 years, one year at a time. In both panels, the entire system design is based on the planning year (e.g. the location of generation assets and the location/capacity of integration options) and then fixed for the other years.

\section{Discussion and insights for policy makers and planners}

In this study we have used a methodology that soft-links a long term whole energy systems model to a high spatial and temporal resolution power systems model to understand how the planning and operation of future VRE focused power systems in Great Britain are affected by inter-annual weather variability. A few caveats of our study need to be highlighted: Our study focuses on the supply side. Demand side measures apart from load shedding are not modelled but would be of value to investigate with a similar approach. The model has a simplified representation of system security (i.e. system adequacy) and grid representation and not all costs such as start-up costs and distribution line upgrades are represented. To keep the model computationally tractable we opt for a linear formulation (we do not represent unit commitment) that solves in a feasible time frame given the number of runs we perform.

We demonstrate the importance of considering inter-annual variability as it has significant impacts on the design and operation of systems with medium and, in some respects even more so, high shares of VREs. We show that inter-annual weather variability drives a spread in system LCOE, caused by both different optimal total investment and operational costs depending on weather year, and $\mathrm{CO}_{2}$ emissions which increases at higher renewable penetration. Furthermore, planning a system based on a decade's worth of variability at once leads to higher total costs and emissions in each individual year of that period than would be expected from the optimal system designs when those years are considered individually.

The key insight here for national policy makers attempting to understand how to achieve long term economy wide decarbonisation targets, such as that mandated by the UK's Climate Change Act, by transitioning to highly renewable electricity system is that modelling analyses which use single weather years may give misleading results. We show that, because such studies neglect the extensive range of weather variability a real world system would experience, they may design systems which are operationally inadequate and fail to meet carbon targets in line with the long term decarbonisation of the whole energy system. Furthermore, depending on the weather year chosen, they may also underestimate the costs of meeting emissions targets with the potential extent of the underestimate growing at higher shares of renewables. While we have focused on the UK, the need for such analyses to consider multiple weather years may be extended to other VRE reliant power systems that experiences significant inter-annual weather variability and do not have access to additional, cost effective flexibility (e.g. on the demand side). This caveat is important to these insights and therefore future work should look to assess the impact of inter-annual weather variability on systems with additional flexibility. From a policy perspective, it is clear that if high shares of VREs are to be a considered an option for the long term decarbonisation of the whole energy system, the implications of inter-annual weather variability must be considered.

For energy planners our results indicate which elements of a future power system with high shares of VRE are consistent, and so may be considered "no-regret" investments, and which are sensitive to the variability of the weather from year to year. We observe consistent spatial patterns in the VRE deployment: most solar energy is deployed in the South and wind energy is located around the country to take advantage of high capacity factors in combination with a diversity in timing of production. However, 
the precise amount of capacity located in a particular region is found to be highly sensitive to weather year. While we notice a spread in profitability of the supply options over the weather years we observe a consistent revenue gap for most technologies (see Supplementary Figures 31 and 32). Several indicators of our study show that the benefits of transmission line upgrades move electricity from the North and South/South-West to demand centres are relatively insensitive to weather year: grid reinforcement significantly reduces LCOE and emissions, capacities are significantly higher with larger shares of VREs and the extent and spatial pattern of reinforcement are all consistent across the weather years. On the contrary, the capacities of flexible generation and storage required for VRE integration are sensitive to weather year, however, their typical spatial deployment pattern is less so. It is important to note that in the 10-year continuous run the capacity of flexible generation is higher than in any of the individual years. Flexible generation, which has low capital and high marginal costs, is used to cover peaks in demand and its utilisation is highly sensitive to the weather year (as can be seen from the inter-annual variability in emissions).

To conclude, our findings show that, if power systems with high shares of VREs are to play a key role in the long term, global decarbonisation goal set out by the Paris Agreement, modellers and decision makers alike must account for their particular system's sensitivity to inter-annual weather variability.

\section{Methods}

\section{highRES model description}

The high spatial and temporal resolution electricity system model (highRES) minimises power system costs to meet hourly demand subject to a number of technical constraints HighRES optimizes the dispatch and locations of power plants as well as the investment and locations of VRE integration options. Other outputs from highRES are total annuitized power system costs, generation per source and emissions. Integration options represented in highRES are flexible generation (modelled as an open cycle gas turbine), electricity storage (modelled as a NaS battery) and high voltage transmission grid reinforcement. Constraints are that supply must match demand in every hour and technical constraints describing the ramping of power plants, storage operation, the flow of electricity via the transmission grid and safety margins. Here, we opt for a linear model with simplified technical complexity but with high spatial and temporal resolution and multiple weather years at full hourly resolution which we feel is suitable for our objectives.

The strong point of highRES is the good representation of VREs. This consists of two parts: a resource assessment and hourly modelling of generation. First, we perform a literature review helping us to define technical, social and environmental exclusion zones (see Supplementary Note 4): For solar energy we differ between rooftop and ground mounted plants. Using data on the building area we calculate the rooftop area in each grid cell. For ground mounted PV we exclude nationally protected areas, good agricultural land, built up areas and areas with a slope greater than 15 degrees. For onshore wind we exclude nationally protected areas, areas with a slope greater than 15 degrees, cities and a buffer distance around cities of 800 metres, 150 meters around highways and 5 kilometres around airports. For offshore wind energy we include areas up to a water depth of 70 metres. To capture the spatial and temporal variability of VRE production and simultaneously model its interaction with storage, the transmission system and conventional generation at large scales (i.e. at the national or continental level), time series data with sufficiently high spatial and temporal detail and coverage are needed (see Supplementary Note 5). Like a number of recent studies $32,33,35,50-53$, we opt to use state of the art global climate reanalysis and satellite based data, both of which provide a suitable balance between temporal and 
spatial resolution while simultaneously maintaining broad, homogenous temporal and spatial coverage. To derive hourly capacity factors for onshore and offshore wind we use data from NCEP Climate Forecast System Reanalysis (CSFR) $)^{54}$. Data is available both on and offshore on a uniform grid at $0.5^{\circ} \times 0.5^{\circ}(35 \mathrm{~km}$ $\mathrm{x} 50 \mathrm{~km}$ ) resolution. We adopt this as our reference grid for the model (see Supplementary Figure 1). For rooftop and ground mounted PV we use data from the Satellite Application Facility on Climate Monitoring (CMSAF) ${ }^{55}$. We convert the data from 2001-2010 into hourly capacity factor. For this study, renewable capacity factors have been aggregated to the zonal level prior to model execution. As such, highRES decides how much capacity is built in a given zone and that capacity is multiplied by the hourly average zonal capacity factor to get generation.

Demand also varies in time and space. We have performed an analysis to see how weather and electricity demand correlate (see Supplementary Figure 7). In general, we see no correlation between demand and wind speed or solar irradiance but a weak anti-correlation with temperature. As a result of this analysis, we run the model using the respective demand year to the weather year. We therefore scale the hourly electricity demand profile from National Grid for 2002- 2010 (2001 is not available and we use the year 2002 for 2001) using the total electricity demand from UKTM for 2050. We have performed a literature review but could not find any studies suggesting a different spatial demand distribution for 2050 compared to today's. We thus disaggregate the demand profiles down to the 20 Seven Year Statement zones (see Figure 3) used by the National Grid based on shares taken from the National Grid's 2005 GB Seven Year Statement. HighRES matches demand and supply at the zonal level. The zones are connected by a simplified representation of the high voltage transmission grid. At present, following refs ${ }^{20-22}$ who developed a very similar model, highRES currently does not consider electricity demand to be price elastic below the value of lost load (VoLL) of $6000 £ /$ MWh (following refs ${ }^{47,48}$ ), at which point load is shed. This is justified from a short run perspective by ref ${ }^{59}$ which finds the elasticity of electricity demand to be very low. From a long run standpoint, refs ${ }^{20-22}$ argue this is justified if the average electricity prices between model runs do not vary dramatically. In our case, the average electricity price across 50VRE and 80VRE runs varies by $\sim \pm 20 \%$. Furthermore, we would argue that while demand elasticity may somewhat affect the total installed capacity of the flexibility options we consider, it unlikely to significantly impact the spatial sensitivity of VREs to weather year that we observe.

A more detailed model description including the equations, data, assumptions can be found in the Supplementary Notes 2-9.

\section{UKTM model description}

We use the long time horizon model UK TIMES model (UKTM) ${ }^{60-63}$ developed at the UCL Energy Institute as a successor to the UK MARKAL mode ${ }^{19}$. In addition to its academic use, UKTM is the central long-term energy system pathway model used for policy analysis at the Department of Energy and Climate Change (DECC) and the Committee on Climate Change (CCC) ${ }^{64,65}$ UKTM is a linear, bottomup, technology-rich cost optimising model instantiated within the TIMES framework ${ }^{60-63}$. It minimizes total energy system costs required to satisfy the exogenously set energy service demands subject to a number of additional constraints ${ }^{66}$. UKTM contains 16 time slices: 4 seasons and 4 intraday (day, evening, late evening, night) and one region (UK). The model comprises a time period from 2010 (the base year) to 2050 with one model period covering 5 years (represented by one representative year). UKTM represents the entire UK energy system from imports and domestic production of fuel resources, through fuel processing and supply, explicit representation of infrastructure, conversion to secondary energy carriers (including electricity, heat and hydrogen), end-use technologies and energy service demands. Generally, it minimizes the total welfare costs (under perfect foresight) to meet the exogenously given, but price elastic, sectoral energy demands under a range of input assumptions and additional constraints, 
thereby delivering a cost optimal, system-wide solution for the energy transition for the coming decades. A key strength of UKTM is that it represents the whole UK energy system under a given decarbonisation objective, which means that trade-offs between mitigation efforts in one sector versus another can be explored. The model is divided into three supply side sectors (resources \& trade, processing \& infrastructure and electricity generation) and five demand sectors (residential, services, industry, transport and agriculture). All sectors are calibrated to the base year 2010, for which the existing stock of energy technologies and their characteristics are known and taken into account. A large variety of future supply and demand technologies are represented by techno-economic parameters such as the capacity factor, energy efficiency, lifetime, capital costs, operation \& maintenance costs. Moreover, assumptions are also exogenously provided for attributes not directly connected to individual technologies, such as fuel import prices, resource availability and the potentials of renewable energy sources. UKTM tracks all energy flows as well as $\mathrm{CO}_{2}, \mathrm{CH}_{4}, \mathrm{~N}_{2} \mathrm{O}$ and $\mathrm{HFC}$ emissions. In addition to its academic use, UKTM is the central longterm energy system pathway model used for policy analysis at the Department of Energy and Climate Change (DECC) and the Committee on Climate Change (CCC).

\section{Study setup}

For this study we soft-link UKTM with highRES. Comparable studies $26,27,67,68$ use a soft-linking approach with the exception of ref ${ }^{28}$ which hard-links a long-term model with a dispatch model (for a number of representative days per year).

We use UKTM to develop internally consistent, whole energy system scenarios that both meet the UK's Climate Change Act $2008^{42}$, i.e. a reduction of greenhouse gas emissions of $80 \%$ relative to 1990 levels by 2050, and have high penetration of VREs. We run UKTM with an 80\% GHG emission reduction target relative to 1990 levels by 2050 and assume that CCS is not available. We make this assumption for two reasons. Firstly, to ensure UKTM designs a power system, and therefore energy system, with high shares of VREs. Secondly, because of the sizable uncertainty around whether CCS will ever feature at scale, particularly in light of the UK government recently cancelling its $f_{1}$ bn CCS competition. We use this as our first scenario for an internally consistent exemplar of a power system with high shares of VREs for our analysis. As it produces $50 \%$ generation from VREs we call it 50VRE. We then run a second scenario with the same characteristics as the first but also apply a constraint that UKTM must generate a minimum of $80 \%$ of electricity from VRE and name it $80 \mathrm{VRE}$.

We use total electricity demand, fuel prices, generation capacities and the $\mathrm{CO}_{2}$ grid intensity from UKTM as input into highRES: As UKTM is a whole energy system model it sets the boundaries on the electricity system (i.e. determines which sectors get electrified). We combine total annual electricity demand for 2050 from UKTM with historical from National Grid hourly load profiles. We use marginal commodity prices for 2050 from UKTM (i.e. the shadow price) as fuel costs in highRES. We take power generation capacities from UKTM as input into highRES. UKTM decides on the grid CO2 intensity for the power sector in order to reach an overall $80 \%$ GHG emission reduction. Within UKTM there is a parametrisation of flexible generation used to integrate VREs into the system. However, here we discard the capacities of flexible generation suggested by UKTM and let highRES decide. HighRES finds the optimal location for generation capacities as well as the optimal capacities and locations of VRE integration options. Supplementary Figure 10 shows how we soft-link UKTM to highRES.

For the first part of the study we perform 44 different model runs: using the weather data from 2001-2010 each year at a time and for the whole 10 years at once for the two scenarios (50VRE and 80VRE) and two different cases. In the allflex case highRES can invest into all three integration options, in the flex+storage case we fix transmission lines to their current capacities and only allow the model to 
invest into electricity storage and flexible generation. In all runs highRES is restricted from installing any nuclear, biomass or OCGT capacity in Z14 (London). Nuclear is also prevented from being deployed in Northern Scotland.

In order to test how planning for a single year affects system operation in other years we need identify the most weather sensitive system. We therefore run every combination of planning weather year and input weather year for the allflex case of both our VRE generation scenarios ( 90 model runs per scenario). That is, in these runs all capacities (generation, storage and transmission) and their locations are fixed to the planning weather year, for example 2001, while VRE generation and demand come from the remaining years 2002-2010, one year at a time. Here we define system weather sensitivity based on the cumulative number of hours with unmet demand over the remaining nine years. We find 2008 and 2005 to be the most sensitive systems for 50VRE and 80VRE respectively. Furthermore, it is important to note that the much larger fraction of unmet demand hours in the latter scenario is strongly influenced by the interaction between its weather sensitivity and the grid CO2 intensity limit applied to the system. This is because the system's sensitive to weather leads to an increase in the utilisation of flexible fossil generation up to a level permitted by the CO2 intensity constraint and beyond which the demand becomes unmet. We also investigate the impact of reaching long-term emission targets when planning the system based on only one year of weather data. We fix the power system design (capacities and spatial distribution) obtained from each weather year for the allflex cases and run the model using the 9 remaining weather years with the $\mathrm{CO}_{2}$ constraint turned off (90 model runs per scenario) (see Supplementary Figure 30).

A few caveats of our study need to be highlighted: Our study focuses on the supply side. Demand side measures are not modelled but would be of value to investigate with a similar approach. The model has a simplified representation of system security (i.e. system adequacy) and grid representation and not all costs such as start-up costs and distribution line upgrades are represented. To keep the model computationally tractable we opt for a linear formulation (we do not represent unit commitment) that solves in a feasible time frame given the number of runs we perform: 400 with 8760/8784 (leap year) time steps and 4 decadal runs.

Supplementary Figure 11 illustrates the study setup including the number of scenarios, cases, running modes, temporal coverage and number of model runs.

\section{Data availability}

The highRES model and UKTM results are available from the corresponding author upon request. The UKTM model will be made publically available in early 2018. 


\section{References}

1. United Nations Framework Convention on Climate Change. Paris Agreement. (2015). Available at: http://unfccc.int/files/essential_background/convention/application/pdf/english_paris_agreement. pdf.

2. Jacobson, M. Z. Energy modelling: Clean grids with current technology. Nature Climate Change 6, $441-442$ (2016).

3. IPCC. Renewable Energy Sources and Climate Change Mitigation Special Report of the Intergovernmental Panel on Climate Change. (Cambridge Univ Pr, 2011).

4. Huberty, M. \& Zysman, J. An energy system transformation: Framing research choices for the climate challenge. Research Policy 39, 1027-1029 (2010).

5. Mitchell, C. Momentum is increasing towards a flexible electricity system based on renewables. Nature Energy 1, 15030 (2016).

6. OECD. World Energy Outlook 2015. (OECD Publishing, 2015).

7. Williams, J. H. et al. The Technology Path to Deep Greenhouse Gas Emissions Cuts by 2050: The Pivotal Role of Electricity. Science 335, 53-59 (2012).

8. Freris, L. L. \& Infield, D. G. Renewable energy in power systems. (John Wiley \& Sons, 2008).

9. Fusco, F., Nolan, G. \& Ringwood, J. V. Variability reduction through optimal combination of wind/wave resources - An Irish case study. Energy 35, 314-325 (2010).

10. Elliott, D. A balancing act for renewables. Nature Energy 1, 15003 (2016).

11. Braff, W. A., Mueller, J. M. \& Trancik, J. E. Value of storage technologies for wind and solar energy. Nature Clim. Change 6, 964-969 (2016). 
12. Lund, P. D., Lindgren, J., Mikkola, J. \& Salpakari, J. Review of energy system flexibility measures to enable high levels of variable renewable electricity. Renewable and Sustainable Energy Reviews 45, 785-807 (2015).

13. Nahmmacher, P., Schmid, E., Hirth, L. \& Knopf, B. Carpe diem: A novel approach to select representative days for long-term power system modeling. Energy 112, 430-442 (2016).

14. Simoes, S. et al. Impact of different levels of geographical disaggregation of wind and PV electricity generation in large energy system models: A case study for Austria. Renewable Energy 105, 183-198 (2017).

15. Poncelet, K., Delarue, E., Six, D., Duerinck, J. \& D'haeseleer, W. Impact of the level of temporal and operational detail in energy-system planning models. Applied Energy 162, 631-643 (2016).

16. Nicolosi, M., Mills, A. \& Wiser, R. The Importance of High Temporal Resolution in Modeling Renewable Energy Penetration Scenarios. (2011). Available at: https://pubarchive.lbl.gov/islandora/object/ir:154930/datastream/PDF/.../citation.pdf.

17. DeCarolis, J. et al. Formalizing best practice for energy system optimization modelling. Applied Energy 194, 184-198 (2017).

18. Pina, A., Silva, C. \& Ferrão, P. Modeling hourly electricity dynamics for policy making in long-term scenarios. Energy Policy 39, 4692-4702 (2011).

19. Kannan, R. \& Turton, H. A Long-Term Electricity Dispatch Model with the TIMES Framework. Environ Model Assess 18, 325-343 (2012).

20. Ludig, S., Haller, M., Schmid, E. \& Bauer, N. Fluctuating renewables in a long-term climate change mitigation strategy. Energy 36, 6674-6685 (2011).

21. Krakowski, V., Assoumou, E., Mazauric, V. \& Maïzi, N. Feasible path toward 40-100\% renewable energy shares for power supply in France by 2050: A prospective analysis. Applied Energy 171, 501522 (2016). 
22. Merrick, J. H. On representation of temporal variability in electricity capacity planning models. Energy Economics 59, 261-274 (2016).

23. Welsch, M. et al. Supporting security and adequacy in future energy systems: The need to enhance long-term energy system models to better treat issues related to variability. Int. J. Energy Res. 39, 377396 (2015).

24. Seljom, P. \& Tomasgard, A. Short-term uncertainty in long-term energy system models — A case study of wind power in Denmark. Energy Economics 49, 157-167 (2015).

25. Deane, J. P., Chiodi, A., Gargiulo, M. \& Ó Gallachóir, B. P. Soft-linking of a power systems model to an energy systems model. Energy 42, 303-312 (2012).

26. Deane, J. P., Gracceva, F., Chiodi, A., Gargiulo, M. \& Gallachóir, B. P. Ó. Assessing power system security. A framework and a multi model approach. International Journal of Electrical Power \& Energy Systems 73, 283-297 (2015).

27. Deane, J. P., Gracceva, F., Chiodi, A., Gargiulo, M. \& Ó Gallachóir, B. Soft-Linking Exercises Between TIMES, Power System Models and Housing Stock Models. in Informing Energy and Climate Policies Using Energy Systems Models (eds. Giannakidis, G., Labriet, M., Ó Gallachóir, B. \& Tosato, G.) 30, 315-331 (Springer International Publishing, 2015).

28. Després, J. Modélisation du développement à long terme du stockage de l'électricité dans le système énergétique global. (PhD thesis. Université Sité Grenoble Alpes, 2015).

29. Hart, E. K. \& Jacobson, M. Z. A Monte Carlo approach to generator portfolio planning and carbon emissions assessments of systems with large penetrations of variable renewables. Renewable Energy 36, 2278-2286 (2011).

30. Gils, H. C. \& Simon, S. Carbon neutral archipelago - 100\% renewable energy supply for the Canary Islands. Applied Energy 188, 342-355 (2017). 
31. MacDonald, A. E. et al. Future cost-competitive electricity systems and their impact on US CO2 emissions. Nature Climate Change 6, 526-531 (2016).

32. Frew, B. A., Becker, S., Dvorak, M. J., Andresen, G. B. \& Jacobson, M. Z. Flexibility mechanisms and pathways to a highly renewable US electricity future. Energy 101, 65-78 (2016).

33. Pfenninger, S. \& Keirstead, J. Renewables, nuclear, or fossil fuels? Scenarios for Great Britain's power system considering costs, emissions and energy security. Applied Energy 152, 83-93 (2015).

34. Mills, A. D. \& Wiser, R. H. Strategies to mitigate declines in the economic value of wind and solar at high penetration in California. Applied Energy 147, 269-278 (2015).

35. Schmidt, J., Cancella, R. \& Pereira Jr., A. O. An optimal mix of solar PV, wind and hydro power for a low-carbon electricity supply in Brazil. Renewable Energy 85, 137-147 (2016).

36. Nelson, J. et al. High-resolution modeling of the western North American power system demonstrates low-cost and low-carbon futures. Energy Policy 43, 436-447 (2012).

37. National Renewable Energy Laboratory. Regional Energy Deployment System (ReEDS) Model Documentation: Version 2016. (2016).

38. Pfenninger, S. Dealing with multiple decades of hourly wind and PV time series in energy models: A comparison of methods to reduce time resolution and the planning implications of inter-annual variability. Applied Energy 197, 1-13 (2017).

39. Burtin, A., Silva, V. \& EDF Research and Development Division. Technical and economic analysis of the European electricity system with 60\% RES. (2015). Available at: http://www.energypost.eu/wp-content/uploads/2015/06/EDF-study-for-download-on-EP.pdf.

40. ADEME, Artelys, ArminesDemain \& Energies Demain. A 100\% renewable electricity mix? Analysis and optimisation- Exploring the boundaries of renewable power generation in France by 2050. (2016). Available at: http://www.ademe.fr/sites/default/files/assets/documents/renewableelectricity-mix-executive-summary-ademe-201601.pdf. 
41. Cox, E. Opening the black box of energy security: A study of conceptions of electricity security in the United Kingdom. Energy Research \& Social Science 21, 1-11 (2016).

42. Parliament of the United Kingdom. The Climate Change Act 2008. (2008).

43. elementenergy \& De Montfort University. Demand side response in the non-domestic sector- Final report for Ofgem. (2012). Available at: http://www.element-energy.co.uk/wordpress/wpcontent/uploads/2012/07/Demand-Side-Response-in-the-non-domestic-sector.pdf.

44. Sustainability First \& elementenergy. What is the potential availability of DSR? (2012). Available at: https://www.ofgem.gov.uk/ofgem-publications/56996/sustainability-first-and-element-energydemand-side-response-event-autumn-2012-pdf.

45. Schleich, J. \& Gruber, E. Beyond case studies: Barriers to energy efficiency in commerce and the services sector. Energy Economics 30, 449-464 (2008).

46. Zerrahn, A. \& Schill, W.-P. On the representation of demand-side management in power system models. Energy 84, 840-845 (2015).

47. nationalgrid. Long-term Market and Network Constraint Modelling. (2017). Available at: https://www.nationalgrid.com/sites/default/files/documents/Longterm $\% 20$ Market $\% 20$ and $\% 20$ Network $\% 20$ Constraint $\% 20$ Modelling.pdf.

48. Department of Energy and Climate Change. Annex C: Reliability Standard Methodology. (2013). Available at: https://www.gov.uk/government/uploads/system/uploads/attachment_data/file/267613/Annex_ C_-_reliability_standard_methodology.pdf.

49. House of Commons. Energy and Climate Change - Second Report The future of Britain's electricity networks. (2010). Available at: https://publications.parliament.uk/pa/cm200910/cmselect/cmenergy/194/19402.htm. 
50. Becker, S. et al. Features of a fully renewable US electricity system: Optimized mixes of wind and solar PV and transmission grid extensions. Energy 72, 443-458 (2014).

51. Olauson, J. et al. Net load variability in Nordic countries with a highly or fully renewable power system. Nature Energy 1, 16175 (2016).

52. Staffell, I. \& Pfenninger, S. Using bias-corrected reanalysis to simulate current and future wind power output. Energy 114, 1224-1239 (2016).

53. Pfenninger, S. \& Staffell, I. Long-term patterns of European PV output using 30 years of validated hourly reanalysis and satellite data. Energy 114, 1251-1265 (2016).

54. Saha, S. et al. The NCEP Climate Forecast System Reanalysis. Bull. Amer. Meteor. Soc. 91, 1015-1057 (2010).

55. Schulz, J. et al. Operational climate monitoring from space: the EUMETSAT Satellite Application Facility on Climate Monitoring (CM-SAF). Atmos. Chem. Phys. 9, 1687-1709 (2009).

56. Hirth, L. The European Electricity Market Model EMMA. Model documentation. Version 2017-0712. (2017). Available at: http://neon-energie.de/EMMA.pdf.

57. Hirth, L. \& Steckel, J. C. The role of capital costs in decarbonizing the electricity sector. Environ. Res. Lett. 11, 114010 (2016).

58. Hirth, L. The market value of variable renewables: The effect of solar wind power variability on their relative price. Energy Economics 38, 218-236 (2013).

59. Lijesen, M. G. The real-time price elasticity of electricity. Energy Economics 29, 249-258 (2007).

60. Fais, B., Sabio, N. \& Strachan, N. The critical role of the industrial sector in reaching long-term emission reduction, energy efficiency and renewable targets. Applied Energy 162, 699-712 (2016). 
61. Daly, H. E., Scott, K., Strachan, N. \& Barrett, J. Indirect CO2 Emission Implications of Energy System Pathways: Linking IO and TIMES Models for the UK. Environ. Sci. Technol. 49, 10701-10709 (2015).

62. Fais, B., Keppo, I., Zeyringer, M., Usher, W. \& Daly, H. Impact of technology uncertainty on future low-carbon pathways in the UK. Energy Strategy Reviews 13-14, 154-168 (2016).

63. Zeyringer, M., Fais, B., Keppo, I. \& Price, J. The potential of marine energy technologies in the UK Evaluation from a systems perspective. Renewable Energy 115, 1281-1293 (2018).

64. Department of Energy and Climate Change. Impact Assessment for the level of the fifth carbon budge. (2016). Available at:

http://www.legislation.gov.uk/ukia/2016/152/pdfs/ukia_20160152_en.pdf.

65. Committee on Climate Change. The Fifth Carbon Budget The next step towards a low-carbon economy. (2015). Available at: https://www.theccc.org.uk/wpcontent/uploads/2015/11/Committee-on-Climate-Change-Fifth-Carbon-Budget-Report.pdf.

66. Loulou, R. \& Labriet, M. ETSAP-TIAM: The TIMES integrated assessment model Part I: Model structure. Computational Management Science 5, 7-40 (2008).

67. Pina, A., Silva, C. A. \& Ferrão, P. High-resolution modeling framework for planning electricity systems with high penetration of renewables. Applied Energy 112, 215-223 (2013).

68. Deane, J. P., Chiodi, A., Gargiulo, M. \& Ó Gallachóir, B. P. Soft-linking of a power systems model to an energy systems model. Energy 42, 303-312 (2012).

69. National Statistics. Plant capacity: United Kingdom (DUKES 5.7). (2017). Available at: https://www.gov.uk/government/statistics/electricity-chapter-5-digest-of-united-kingdom-energystatistics-dukes. (Accessed: 20th February 2018) 
70. National Statistics. Capacity of, and electricity generated from, renewable sources (DUKES 6.4). (2017). Available at: https://www.gov.uk/government/statistics/renewable-sources-of-energychapter-6-digest-of-united-kingdom-energy-statistics-dukes. (Accessed: 20th February 2018)

71. National Statistics. Power Stations in the United Kingdom (DUKES 5.11). (2017). Available at: https://www.gov.uk/government/statistics/electricity-chapter-5-digest-of-united-kingdom-energystatistics-dukes. (Accessed: 20th February 2018)

72. National Statistics. Electricity: commodity balances (DUKES 5.1). (2016). Available at: https://www.gov.uk/government/statistics/electricity-chapter-5-digest-of-united-kingdom-energystatistics-dukes.

73. National Statistics. Digest of UK Energy Statistics (DUKES): Chapter 5: Electricity. (2017). Available at: https://www.gov.uk/government/uploads/system/uploads/attachment_data/file/633779/Chapter -5.pdf.

\section{Acknowledgements}

This research was supported under the Whole Systems Energy Modelling Consortium (WholeSEM) - Ref: EP/K039326/1. We would like to thank David Brayshaw for his comments at the wholeSEM conference which have improved our analysis, Neil Strachan for his comments on an earlier draft and Andy Moore for fruitful discussions. 


\section{Author information}

\section{Affiliations}

UCL Energy Institute, University College London, WC1H 0NN London, United Kingdom Marianne Zeyringer, James Price, Birgit Fais, Ed Sharp

Copernicus Institute of Sustainable Development, Utrecht University, 3584 CS Utrecht, The Netherlands

Marianne Zeyringer

Institute for Sustainable Economic Development, University of Natural Resources and Life Sciences (BOKU), Vienna, 1180 Vienna, Austria

Marianne Zeyringer

Contributions

M.Z. designed the study with J.P. M.Z. and J.P. have developed the highRES model. B.F. co-built the UKTM model. M.Z. and J.P conducted the highRES model runs. B.F. and P.L. conducted the UKTM model runs. M.Z. conducted the spatial analysis on the capacity potential per technology, E.S. generated the wind time series, J.P. generated the PV time series. B.F. wrote the text on UKTM, E.S. wrote the text on generating wind time series. M.Z. wrote the document with J.P. and they generated figures and tables.

Competing interests

The authors declare no competing interests.

Corresponding author

Correspondence to Marianne Zeyringer (m.zeyringer@ucl.ac.uk) 